\title{
The combination of national music elements and western composition techniques in Liu Xiaogeng's Piano Works_-In the piano works "Sani fantasy"
}

Xianying Zhou

\author{
School of music and dance, Qujing Normal University, Yunnan 655011 \\ 655011@126.com
}

Keywords: Piano Works "Sani fantasy"; writing techniques; performance techniques; Liu Xiaogeng;

\begin{abstract}
Liu xiaogeng is Yunnan Province is well-known local composer, Yunnan minority music culture and art elements was selected in all of his works theme elements, reflecting the strong wind of Yunnan ethnic music and highlight their unique artistic style. Among them, piano works "Sani Fantasia" is Liu xiaogeng in the eighties initial creation of a song has a strong style of Sani Piano Variations, paper mainly on Liu xiaogeng, creation of the Sani fantasy song "of analysis and research, from Yunnan elements of folk music and western composition techniques of vision on the creation of Liu xiaogeng this first piano works with national characteristics, creation background theme, artistic features and formal structure of multi-dimensional analysis, and thus to explore the inheritance and protection of Yunnan ethnic style of piano music and ethnic music in Yunnan relationship.
\end{abstract}

\section{Introduction}

Liu xiaogeng, Yunnan Province is well-known local composer, Yunnan minority music culture and art elements was selected in all of his works theme elements, usually the modern composing techniques and the Chinese traditional elements combined, reflects the rich Yunnan folk music wind and is showing its own unique artistic style. The piano piece "Sani Fantasia" is Liu xiaogeng in 1980s mid creation a song with strong Sani style of Piano Variations, had been scheduled for 1997 Hong Kong Piano Competition repertoire, in the whole of China has been widely recognized and promotion.

\section{1. "Fantasia" creation background}

In the mid-1980s, in Yunnan Art Institute is nearing completion of academic Liu xiaogeng in Yunnan this unique treasure of Feng Shui growth for more than 20 years, when outside the composer began in Yunnan Folk Song adapted in such a way as to the creation of piano music, Wang Jianzhong "cloud five pieces of folk songs of the South", Zhu Jianer's five Yunnan Folk Songs ", Li Yinghai" Creek drips water "and so on, these piano works have been very quickly becoming popular piano works in the country, by the general music education professionals and the broad masses of the people love and promotion. Liu Xiaogeng was as a composer professional graduate student, Yunnan traditional folk music culture has already deep into his blood, because love on the road with Liu Xiaogeng piano music in addition to bell piano feeling is subject to the creation of thought influence, as a full blooded Yunnan music professional graduates young Liu Xiaogeng, the more urgent need to show themselves by learning the composing skills to express his love for Yunnan folk music culture. The piano by this instrument, he also wanted to create a 'sunburn population of Yunnan style piano works, but not just rely on the Yunnan folk songs with such a structure adapted to that is more about how to put the piano timbre to acme, through their own creative language, a blend of western music, to create To make more appropriate Yunnan 
national style and more innovative for the public to accept the love of piano works.

In many Yunnan folk songs, folk song which attracted Liu Xiaogeng's vision, then by Fan Yu and co wrote "song writer Mai Ding guests from afar you stay" this folk song have become known to every family of Yunnan folk songs, Liu Xiaogeng played the song on the piano, with a composer keen he found the song's Melody feature already contains all the principal and subordinate genera, this trait is very harmony, Sani big Sanxian flavor, and is suitable for the piano range to express, after repeated processing, modification, two degrees of creation, "Sani Fantasia" this is the first known piano it breeds born the works.

\section{2. "Fantasia" Sani ethnic music elements}

Yi is the largest ethnic minority population in Southwest China, is also a versatile, national dance, Yi Sani nationality belongs to a branch, mainly living in Honghe Shilin County, in the long river of history, ancestors of Sani use their own spoken and written languages left a large number of literature, recording. All of these have become the important part in the traditional culture of the Yi nationality, such as literature is the famous long narrative poem Ashima, in terms of art such as the traditional songs and dances the Sani big Sanxian dance "is also the Sani traditional folk dance Dao. In the traditional dance of Sani always Dudu showing a bright and smooth rhythm feeling, and their song and dance and music is often accompanied by blowing, playing, singing, dancing, with music and dance art characteristics of integration, which is the Yi national minority folk music and dance characteristics. Liu xiaogeng creation of the song "Sani Fantasia", draw the Sani ethnic group in Yunnan unique musical elements and the characteristics of national music, and the use of novel creation technique, the Yunnan Yi Sani unique native music traits ascribed to the piano this is widely popular Western musical instrument, the Yi People's traditional music and dance in a new burst out of the era of the rhythm of life.

\section{Music elements}

Yi's music varied and colorful, different regions, different branches of the Yi folk song has the different characteristic, Sani music in the Mixolydian scale major pentatonic scalescale mainly showed two basic types, of which a special scale for "three degrees" of the three sound framework and add phonetic change style, such as shown in the figure.

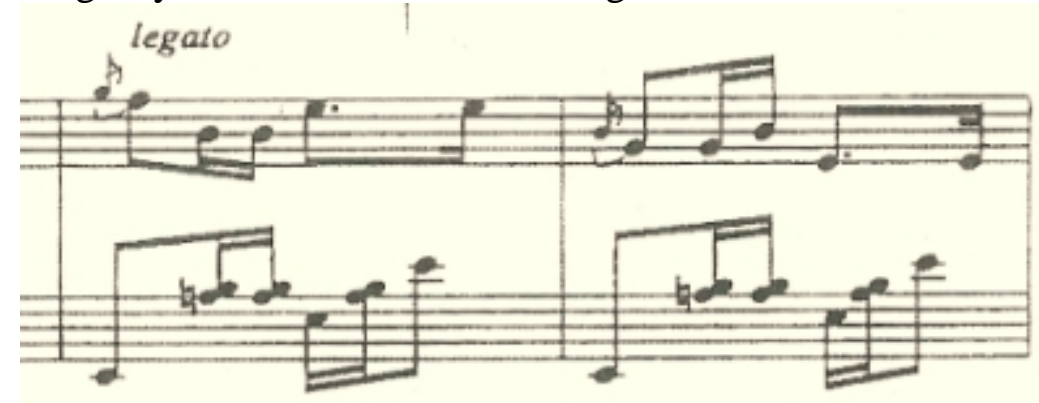

A two bar theme used the Yi traditional "do Mi sol" such a sound framework, loud melody representative soprano flute melody, low voice on behalf of the chords shifting Sanxian and dance steps. The stereo image expressed the Yi people in Tiao brisk lively dance scene.

Another feature is "three tone scale frame four or five degrees" and modified type, as shown in figure: 


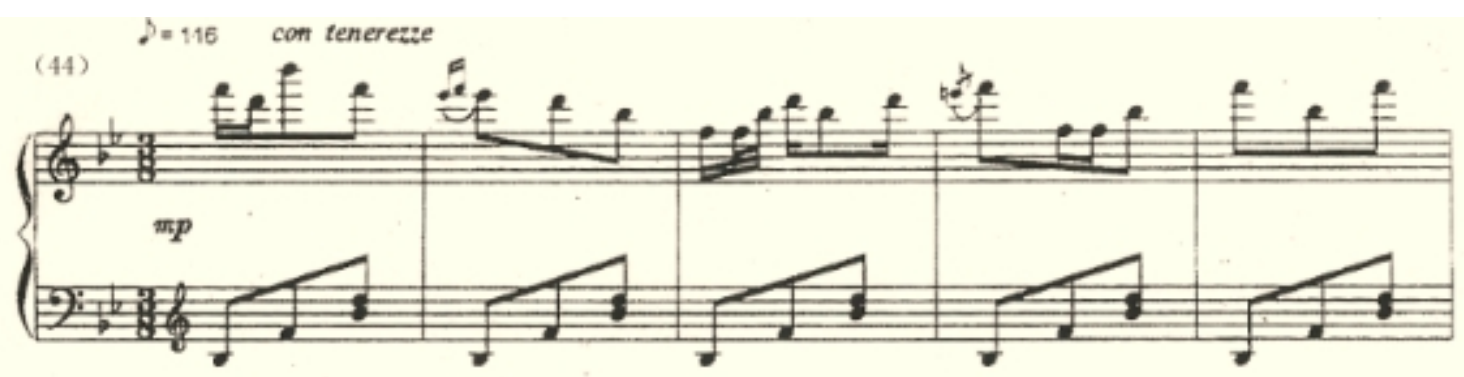

Theme of the first section use "Sol do re" such a sound framework and inflexion style, has the characteristics of typical Sani music.

From the rhythm, the music of the Yi people often appear before the short after long rhythm type, as shown in figure:

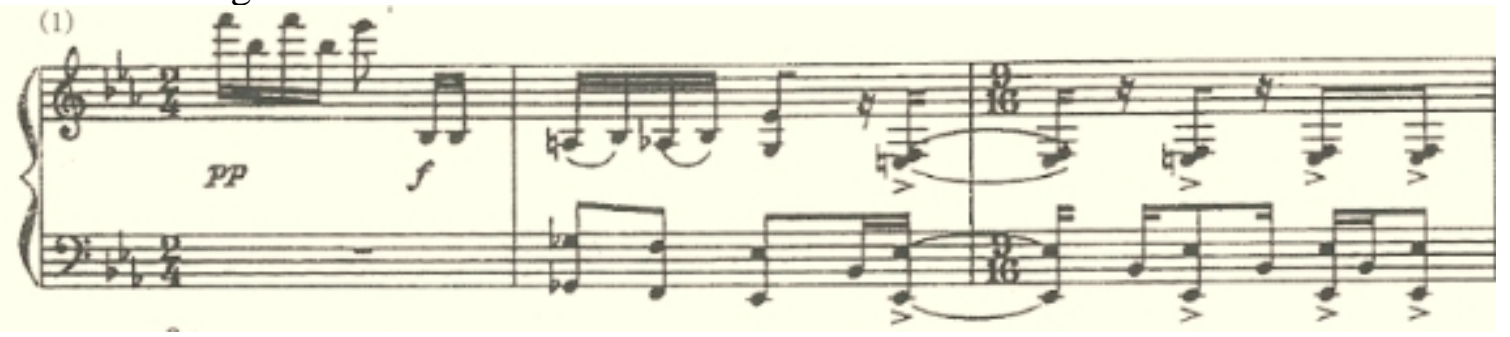

From the second section second to third sections, use the short long before after the rhythm, the rhythm of music enhancement. From the beats in the creation of music, Sani nationality musical style of music usually used more this beat mix, showing a vibrant music, also weakened the repetitive melody bring2/4、3/4、4/4、5/4、6/8、9/8 the sense of monotony. Such as "Sani fantasy" uses the rhythm of the beat, introduction by $2 / 4$ of the first section to take the third section transformation for 9 / 16, that is in line with the Yi peculiar to the music rhythm feeling, also meet the peculiar to Western jazz music rhythm, enhance the rhythm of the music, as well as modern composing technique provides a new thinking and space.

\section{Dance elements}

Sani people in a festive, especially wrestling, bull fighting and "tiaoyue" lunar June 24, "Torch Festival". The Yi people throughout history, "Tiao" and the torch festival have very deep origin, the annual Lunar June October 24 positive Yi Sun Li Yang. At this time the dealer mature, pest birds disaster, the ancestors of the Yi to energize the power to protect themselves. The torch festival of the ancient, specialized fixed Shanchang cattle heaven, because from the beginning of the next day, the sun to the south around, Yin Qi between heaven and earth to rise, the days are short and the nights are long, people will give fire as the sun god farewell, all through the night the moon dance. The dance style is the most characteristics of the pace of three step two bombs, waving walk three steps and clap stomp leg kicking two, a five beat a group of rhythm. In choosing the topic theme in fantasy is a slow "Tiao" tone can be divided into harmonic fast transfer of two slow call 3 / 8 beat, each section clapped their hands, and quickly call 2 / 4 beat, each section clap twice, the men carrying three $\mathrm{Hu}$, flute, erhu, four string dance, dance is relatively simple, the body slightly forward tilt, take a step, and clapping, also known as "cross fibrillation step", "Sani" with such tempo music follows the "distance guest please stay" theme and beat, Vice Minister of the slow call 3 / 8 beat, and before the cheerful rhythm contrast, very beautiful quiet. 


\section{"Sani Fantasia" creative techniques}

\section{"Sani Fantasia" musical structure}

Liu xiaogeng, writing of the Sani Fantasia "is a song omitted the Deputy Ministry of sonata form structure. Here is a selection of the western traditional" Fantasia "writing techniques, also used the sonata form's structure, writing, sonata form is in two in materials and modes of the existing contradiction, and contact the unified theme based, in the music of the positive development, and finally reproduced unified a large musical structure.

Analysis of the following form

Sonata form:

\begin{tabular}{|c|c|c|c|c|c|c|c|c|}
\hline $\begin{array}{c}\text { A musical } \\
\text { structure }\end{array}$ & Primer & \multicolumn{3}{|c|}{ Show the Department } & \multicolumn{2}{|c|}{$\begin{array}{c}\text { Development } \\
\text { Department }\end{array}$} & playback & The end \\
\hline $\begin{array}{c}\text { The two stage } \\
\text { musical structure }\end{array}$ & & $\begin{array}{c}\text { The } \\
\text { main } \\
\text { part } \\
\text { conne } \\
\text { ct }\end{array}$ & $\begin{array}{c}\text { Vice } \\
\text { Minister }\end{array}$ & & connect & & \\
\hline $\begin{array}{c}\text { Number of } \\
\text { starting and } \\
\text { ending sections }\end{array}$ & $1-13$ & $14-38$ & $39-43$ & $44-74$ & $75-133$ & $134-136$ & $137-163$ & $164-185$ \\
\hline modal tonality & & bE & & b B & & & b E & \\
\hline
\end{tabular}

The main theme of the Piano Department chose 《guests from afar you stay $》$ this folk song tone down $\mathrm{E}$ Gong mode, rhythm is 2/4, full of warm and cheerful mood, and vice minister of the theme of the main selection under the Ministry of sound material, and beat into 3/8 beats. Tonality into E mode is lowered B function of palace palace mode, music mood quiet and graceful, after 75 sections to 133rd sections for music, here is a selection of the main Department of music materials, variations in expansion mode, makes music character, image becomes more vivid, after 3 a section of the connection into the omission of the vice Ministry of reproduction Department, the Department is still the main melody of music material, but about the accompaniment into sixteen note flow movement, although using the same music material, but Compared with the previous music style, the main part becomes more lyrical. End of writing is the use of the expansion material development to writing. During the application of the writing techniques in modern music works, when the material has expanded, tonal form your own layout rules, contains a series of unstable harmonies and away the modulation, finally came to an end in B flat tone, curl in the melody, which is start to start function deepening, has become the end of the continuation of the unstable function.

\section{"Sani Fantasia" musical elements with western composing techniques integration}

In Fantasia of Sani, Liu xiaogeng, in addition to the choice of national music elements, also joined the western composing technique, for example, ragtime rhythm, west of diacritical system. These may be to become very lively and very modern music works, also more accord with Yunnan Yi their music unique law system and performance form. Such as music of the introduction part, the author chose the Western ragtime rhythms, with second degree of the audio to form Yi drum rhythm sense of rhythm and sense, as shown in Figure: 


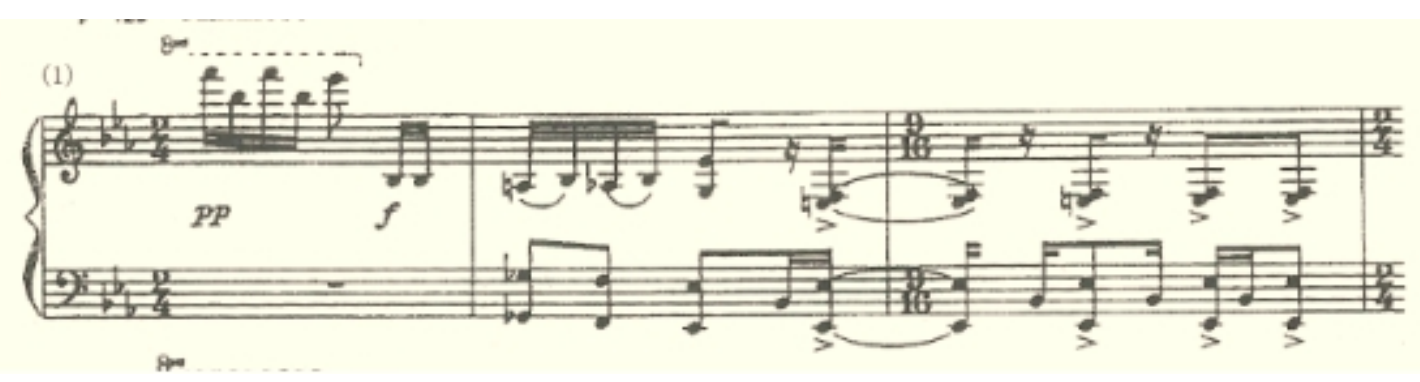

After the introduction in the second section, into the expansion of Western ragtime rhythm, let the music with a greater sense of rhythm, as plucked string instrument of the big Sanxian sweeping strings of feeling, and as minority drum sound. Fantasia of Sani Deputy Ministry of melodic material chosen and subordinate sound reflection structure, from the music of color and form with the principal part form a contrast. As graph;

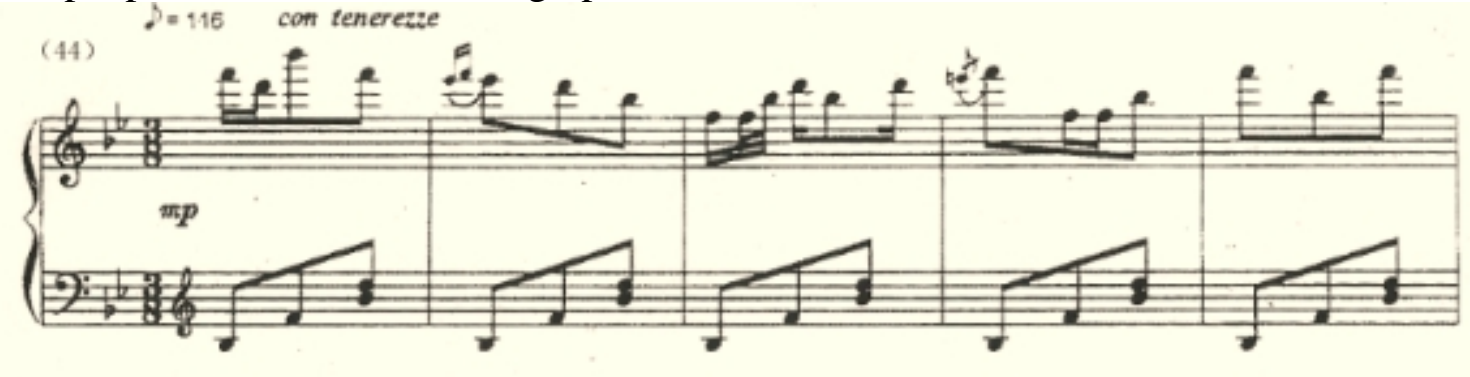

Tunes by before bright and cheerful $\mathrm{E}$ flat palace mode into the gentle and quiet in B flat features adjustable, left hand accompaniment by before the rhythm rhythm into uniform flow of 3 / 8 beat, and before the cheerful rhythm formed a contrast.

Fantasia of Sani development department is selected and music material, into the continuous development and change, die, and joined the western modern composing method in a large number of inflection, the development way of writing both other folk song adapted piano works for single music elements, through a simple music material to change the development, which makes the work more perfect and unified, and content and form become more rich and colorful.

One, and the form content becomes more rich and colorful.

In Fantasia of Sani, Liu xiaogeng still use piano unique sound to imitate drum sound, imitate the voice of the Yi people plucked string Sanxian, as shown in Figure:

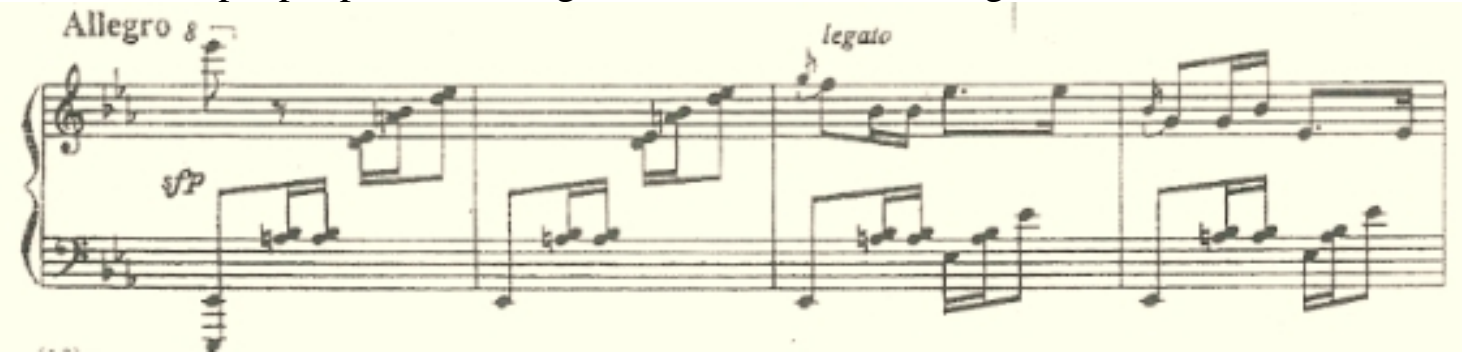

The left hand first started $x$ such jumping rhythm, $\times \times \times \times \times \times$ the rhythm of this writing represents a kind of style of Mr. Xiao Geng Liu's common characteristic of its rhythm is different from the previous traditional rhythm, a remake of the first tone should be slightly emphasized (Gen Yin) and the last sound (i.e. the beginning and at the end of the two eight note), this special rhythm belongs to Sani nationality unique dance rhythm, represents the sound and rhythm of the string Sanxian type, in the author's creation concept, it is not only a piano melody melody representation and musical instruments, its broad range can imitate any sound for example, through a special process to the piano and voice constitute a piece of music, for example the use of piano sound to 
imitate the wind, the rain, what is sometimes the only in the creation of the author Only the piano as a drum, with the percussion sound to heighten the whole sound effects, "Sani fantasy" in some special rhythm interval processing has also become a continuation of the future of the Liu xiaogeng creative style and retention.

From the above analysis, we can see Liu xiaogeng is a has been in quest will melt the trinity of the Yunnan ethnic music essence, the spirit of the times and the composer himself the artistic personality of Yunnan local composer, he who reflects the maturity of the composer has two important signs: one is to establish their own personalized musical language and musical style; another one is to create reflect the personalized style of music works. The piano works are belong to the writing of his youth, showed his unique style of music, and he has been trying to do things is through his creation to the music culture in Yunnan promoted, let Yunnan ethnic music has become the music world, let more and more people love, acceptance, also in order to convey the essence of Yunnan People's spiritual and cultural. And for the future more vivid and lively, highlighting his temperament style of all kinds of works laid the cornerstone.

\section{References}

[1] Zhang Xingrong: "the original ecological folk music of Yunnan", Beijing: Central Conservatory of Music press, 2006.

[2] Zhou Kaimo: Yunnan national music theory, Yunnan: Yunnan people's publishing house, 2007.

[3] Li Meng: "music form analysis", Chongqing: Southwestern Normal University press, 2007

[4] 2008 "solution Junran Axi tiaoyue and Sani big Sanxian dance", [J]. Journal of Central Conservatory of Music, 2008.

[5] The contemporary echo of the Yunnan folk song: a review of the writing style of Liu Xiaogeng's chorus [J]. Sichuan drama. 2013. 